\title{
Neurological manifestations of COVID-19: available evidences and a new paradigm
}

\author{
Fatima Khatoon $^{1} \cdot$ Kartikay Prasad $^{1} \cdot$ Vijay Kumar $^{1}$ (D) \\ Received: 16 June 2020 / Revised: 17 July 2020 / Accepted: 14 August 2020 / Published online: 24 August 2020 \\ (C) Journal of NeuroVirology, Inc. 2020
}

\begin{abstract}
The recent pandemic outbreak of coronavirus is pathogenic and a highly transmittable viral infection caused by Severe Acute Respiratory Syndrome Coronavirus 2 (SARS-CoV2). In this time of ongoing pandemic, many emerging reports suggested that the SARS-CoV-2 has inimical effects on neurological functions, and even causes serious neurological damage. The neurological symptoms associated with COVID-19 include headache, dizziness, depression, anosmia, encephalitis, stroke, epileptic seizures, and Guillain-Barre syndrome along with many others. The involvement of the CNS may be related with poor prognosis and disease worsening. Here, we review the evidence of nervous system involvement and currently known neurological manifestations in COVID-19 infections caused by SARS-CoV-2. We prioritize the 332 human targets of SARS-CoV-2 according to their association with brain-related disease and identified 73 candidate genes. We prioritize these 73 genes according to their spatiotemporal expression in the different regions of brain and also through evolutionary intolerance analysis. The prioritized genes could be considered potential indicators of COVID-19-associated neurological symptoms and thus act as a possible therapeutic target for the prevention and treatment of CNS manifestations associated with COVID-19 patients.
\end{abstract}

Keywords COVID-19 $\cdot$ SARS-CoV-2 $\cdot$ Neurological damage $\cdot$ Gene prioritization $\cdot$ Gene enrichment

\section{Introduction}

The outbreak of novel coronavirus, known as SARS-CoV-2, has challenged the healthcare community around the world. The ability of high infectivity and transmissibility even during the asymptomatic phase resulted in rapid transmission of the virus around the globe, leading to a pandemic situation. The COVID-19 pandemic is of a scale not seen since the 1918 influenza pandemic. The first case of COVID-2019 was founded in Wuhan, China, on December 8, 2019 (Thompson 2020). As of mid-week, of July, 2020, reported cases of COVID-19 surges past 13.8 million around the world and deaths surpasses over 59 lakhs. The clinicians and pathologists realized that even though the lungs are main target, the viral infection can spread to other organs such as heart, bloodvessels, kidney, gut, and brain (Wadman et al. 2020).

Vijay Kumar

vkumar33@amity.edu

1 Amity Institute of Neuropsychology \& Neurosciences, Amity University, Noida Uttar Pradesh 201303 India
The COVID-19 is a single-stranded positively sensed RNA virus, consisting of $26-32 \mathrm{~kb}$-sized genome. The average diameter is $100 \mathrm{~nm}$, spherical, or oval-shaped. The rate of recombination is up to $25 \%$ and is externally covered by a crown shaped like spike (S) proteins, which also can mutate frequently (Wrapp et al. 2020). These characteristics illustrate the adaptability of the virus to change its infectivity over time. The angiotensin-converting enzyme 2 (ACE2) receptors, which normally helps to regulate blood pressure, is abundantly expressed in the lungs. The spike proteins of the COVID-19 bind to ACE2 receptors to invade the cell and develop the infection (Yan et al. 2020). Once the virus controls the host's cell machinery, it starts making countless copies of itself for infecting other cells.

The COVID-19 predominantly affects the respiratory system; however, there are plentiful numbers of evidences suggesting that SARS-CoV-2 can be neuroinvasive, resulting in neurological complications (Troyer et al. 2020; Gklinos 2020; Leonardi et al. 2020). The literature search through the Dimension, a scholarly search database (https://www. dimensions.ai/), we found over 1500 publications related to the search term "COVID-19 and Nervous System." 
A recent updated review by Ellul et al (Ellul et al. 2020) showed increasing evidences of the neurological manifestations in COVID 19 patients; about 901 patients of COVID 19 with the neurological manifestations have been reported. Although the neurological complications are rare in other corona viruses, the minimum frequency of CNS and PNS complications ranged from $0.04 \%$ for SARS to $0.20 \%$ for MERS and $0.05 \%$ for SARS to $0.16 \%$ for MERS respectively (Ellul et al. 2020)

A systematic updated review by Whittaker et al. (Whittaker et al. 2020) discussed neurological manifestations of COVID19 by summarizing 31 studies. These studies reported manifestations like Guillain-Barre syndrome (GBS), headache, olfactory dysfunction, and acute cerebrovascular accidents.

In early March 2020, the Beijing Ditan Hospital reported the first case of viral encephalitis in COVID-19 patients, affecting the central nervous system (CNS). The researchers confirmed the presence of SARS-CoV-2 in the cerebrospinal fluid (CSF) by genome sequencing (Xiang et al. 2020). Subsequently, another case of viral encephalitis was reported in Japan where SARS-CoV-2 was identified in the CSF in the absence of nasopharyngeal positivity, suggesting the possibility of direct infection or indirect routes of transmission such as hematogenous path (Moriguchi et al. 2020). A retrospective series of 214 consecutive hospitalized COVID-19 patients spanning from January 16, 2020, to February 19, 2020, from Wuhan described the neurological manifestations of patients (Mao et al. 2020a). A total of 126 (58.9\%) of 214 patients with COVID-19 had non-severe infection, while $88(41.1 \%)$ patients had severe infection based on respiratory status. The most common symptoms at onset of illness were fever $(61.7 \%)$, cough $(50.0 \%)$, and anorexia (31.8\%). Seventyeight $(36.4 \%)$ patients were found to have neurologic manifestations. These neurologic manifestations were classified as CNS (24.8\%), PNS (8.9\%), and skeletal muscle injury (10.7\%). In patients with CNS manifestations, the most common reported symptoms were dizziness (16.8\%) and headache $(13.1 \%)$, whereas in patients with PNS symptoms, the most common reported symptoms were taste impairment (5.6\%) and smell impairment (5.1\%). Besides these, other disorders were analyzed including hypertension, diabetes, cerebrovascular disease, and malignant and chronic kidney diseases. In a French case series of 58 intensive care patients with COVID-19, 69\% were encephalopathic with agitation or confusion, including $67 \%$ with corticospinal tract signs. Thirtythree percent who had been discharged had a dysexecutive syndrome (Helms et al. 2020a; Helms et al. 2020b).

In the first 3 weeks of the exponential phase of the pandemic, UK-wide surveillance system, (CoroNerve) reported 153 COVID-positive cases with a median age 71 (range was 23-94) years. Out of these 153 cases, 77 (62\%) had a cerebrovascular manifestation including, 57 (74\%) ischemic strokes, nine (12\%) intracerebral hemorrhages, and one CNS vasculitis. The second most common group, 39 (31\%) had altered mental status, including $16(41 \%)$ with encephalopathy of those, 7 (44\%) had encephalitis. The remaining 23 (59\%) had a psychiatric manifestation, including $10(43 \%)$ with psychosis, 6 (26\%) neurocognitive (dementia-like) syndrome, and $4(17 \%)$ an affective disorder (Varatharaj et al. 2020).

Recently, a systematic review and meta-analysis by Rogers and colleagues (Rogers et al. 2020) showed the neuropsychiatric manifestations of SARS- and MERS-CoV during different illness stages. The symptoms like insomnia (41.9\%), anxiety $(35.7 \%)$, memory impairment $(34.1 \%)$, depression (32.6\%), and confusion (27.9\%) were observed during acute illness. During post-illness stage, sleep disorder (100\%), traumatic memories (30.4\%), fatigueness (19.3\%), memory impairment $(18.9 \%)$, irritability $(12.8 \%)$, anxiety $(12.3 \%)$, insomnia (12.1\%), and depression (10.5\%) were commonly observed. Moreover, there was evidence for delirium (confusion) in $65 \%$ of intensive care unit patients and altered consciousness in $21 \%$ of patients who subsequently died in another study (Rogers et al. 2020).

A very recent study showed the neuroinvasive nature of the virus and the likely routes of transmission to the CNS through transmission electron microscopy of the brain tissue of a 74year-old male COVID-19 patient (Paniz-Mondolfi et al. 2020). The studies clearly showed the presence of 80 to $110 \mathrm{~nm}$ viral particles in the frontal lobe, and in small vesicles of endothelial cells. Also, the blebbing of viral particles in or out of the endothelial wall indicates the viral entry or exit across the brain microvascular endothelial cells into the neural system. Moreover, neural cell bodies exhibited enlarged cytoplasmic vacuoles containing enveloped viral particles with distinct stalk-like projections. Also, autopsy reports have revealed brain tissue edema and partial neuronal degeneration in dead patients (Xu et al. 2020). Furthermore, in India, a 72year-old patient showed up with acute onset cerebellar ataxia followed by encephalopathy. When diagnosed, the results showed that he was suffering with SARS-CoV-2-induced pneumonia, and revealed that the neurological manifestations could be the earlier symptoms of COVID-19 (Lahiri and Ardila 2020).

\section{Routes to SARS-CoV-2 infection in the nervous system}

The neuroinvasive nature of coronavirus has been illustrated as common features. The SARS-CoV and SARS-CoV-2 showed the greater similarity; it is quite possible that they also possess a similar potential. The neurotrophic viruses may enter the brain by multiple routes including direct and indirect pathways (Fig. 1). 
Fig. 1 The mechanism of coronavirus infections and neurological damage caused by SARS-CoV-2. The coronavirus can enter the nervous system directly through the olfactory nerve, blood circulation, ACE2 in brainstem, immune injury, and neuronal pathways, resulting in neurological disorders. The COVID-19 infection in the gastrointestinal tract could use the enteric nervous system (ENS) and its sympathetic afferent neurons to reach the CNS

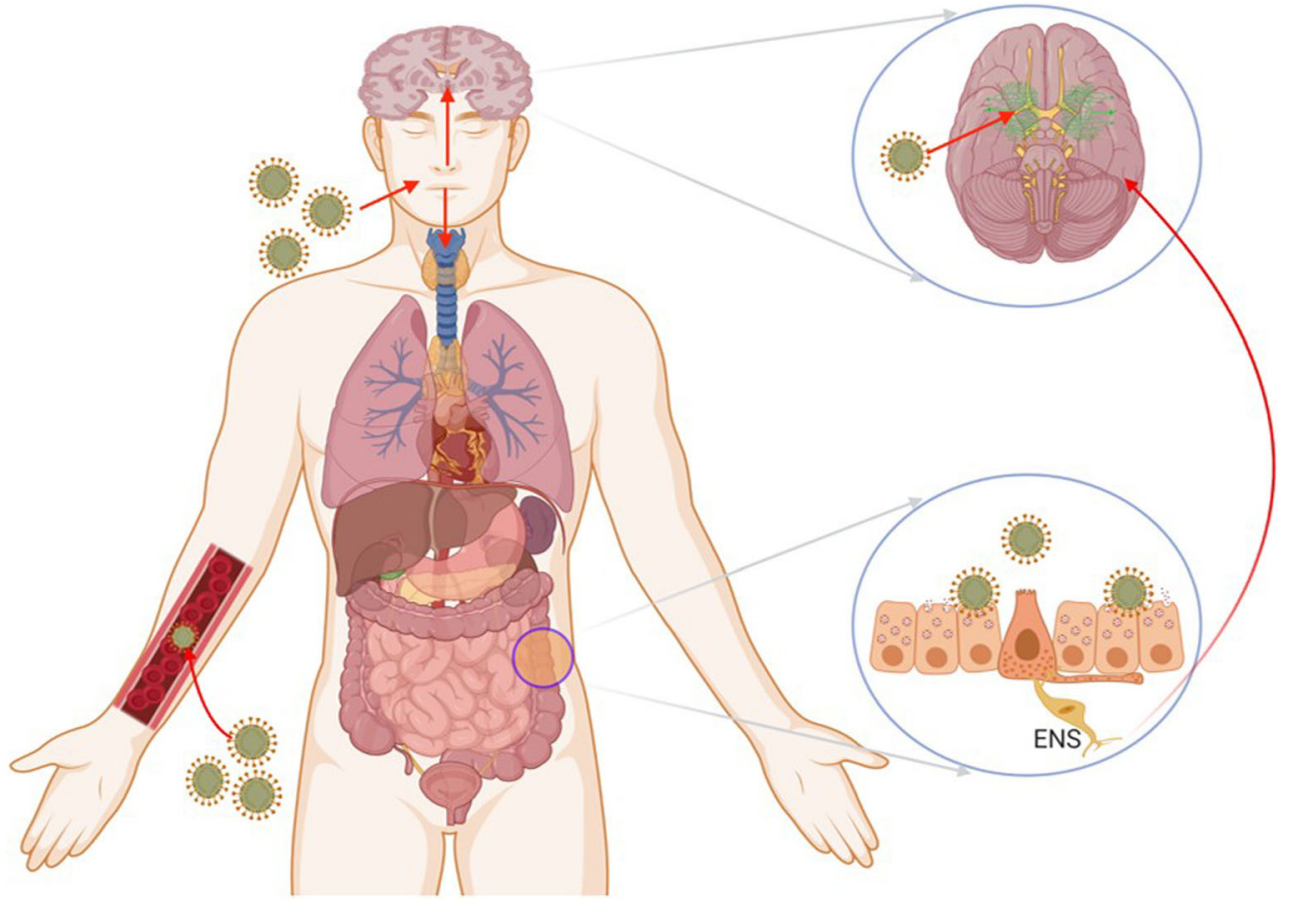

The virus gains entry by infecting endothelial cells of the blood-brain-barrier, epithelial cells of the blood-CSF barrier in the choroid plexus, or using inflammatory cells to enter the CNS (myeloid cell trafficking). More importantly, the virus can utilize retrograde axonal transport to reach the CNS. Retrograde axonal transport may occur through the olfactory, respiratory, and enteric nervous system networks. After infecting the nasal cells, the virus can invade into the brain directly, possibly through the olfactory bulbs and rapidly extend to specific brain areas such as the thalamus and brainstem, causing inflammation and demyelinating reaction (Bohmwald et al. 2018). It explained that viral infection may infect the nasal passage and causing the disruption of senses of smell and taste. The increasing evidence shows that COVID-19 may gain access to the CNS through a synapse-connected route after invading peripheral nerve terminals of the respiratory network (Li et al. 2020).

Also, the SARS-CoV-2 could utilize the sympathetic afferent neurons of the enteric nervous system (ENS) to gain the access to CNS during infection in gastrointestinal tract (Toljan 2020). Epidemiological surveys on COVID-19 illustrated that the average time from initial symptoms to the intensive care took around 8 days, and the window period of the virus is sufficient for invading and devastating the medullary neurons (Wang et al. 2020). The conditions may get worsened for COVID-19 patients with pre-existing neurological disorders.

The receptor for SARS-CoV-2 to invade the host's cells is ACE2. ACE2 also contributes majorly in controlling vasoconstriction and blood pressure and are associated with cardiovascular functions. ACE2 expresses in number of cell types including the lung, kidney, intestine, and brain. In the brain,
ACE2 also expresses in brainstem nuclei, and plays role in cardio-respiration regulation (Baig et al. 2020). Thus, the interaction of spike proteins with ACE2 receptors in brainstem nuclei can also accompany respiration problems in COVID19 patients. A medical graduate student from Wuhan University, a COVID-19 survivor, complained about natural breathing that she had to stay conscious and must breathe continuously and actively during the intense care. Otherwise, if she fell asleep, she might die because she had lost her natural breath (Li et al. 2020). The virus may spreadout trans-neuronally to distant brain targets, same as the other neurotropic viruses (McGavern and Kang 2011). Even when the virus moves from lungs to the medulla and travels from synapses to cardiorespiratory neurons in the brain, the infection of cardiorespiratory neurons may terminate the function properly by inhibiting spontaneous breathing (Wu et al. 2020). The ACE2 receptors are also expressed in capillary endothelium. The virus by interacting with ACE2 may damage the $\mathrm{BBB}$ and gain access to the CNS via attacking vascular system (Wu and Yang 2020).

The virus can also reach the cerebral circulation via blood circulation. The sluggish circulation of blood within mircocirculations may facilitate the binding of spike protein with ACE2 receptors expressed on the capillary endothelium. Furthermore, the damaging of endothelial lining due to increased viral particles can facilitate the viral access to brain. Once the virus reaches the neuronal tissues, spike proteins interact with ACE2 receptor expressed on neuronal cells, and starts the cycle of viral replication, accompanied by neuronal destruction without substantial inflammation, which has been seen with cases of SARS-CoV in previous years (Baig 
et al. 2020). A report of Dutch COVID-19 patients showed the abnormal clotting of blood. The blood clots can break and move to different parts of the body; it can even land in lungs and block vital arteries and cause pulmonary embolism, which is reported as vital in COVID-19 patients. The blocking of arteries in brain due to blood clots can cause stroke (Wadman et al. 2020).

\section{Neurological manifestations}

The neurological manifestation and complications can be divided into central nervous and peripheral nervous systems.

\section{CNS manifestations}

The central nervous system manifestations include epilepsy, ataxia, encephalitis, impaired conscious, Acute Hemorrhagic Necrotizing Encephalopathy (ANE), and headache. Encephalitis refers to the inflammatory lesions in the brain, which includes nerve tissue lesions and neuronal damage. Moriguchi and colleagues reported a 24year-old patient, in Japan, associated with encephalitis who was admitted in hospital with fever followed by seizure and unconsciousness (Moriguchi et al. 2020). Wong et al. reported another case from the UK of a man, 40 years old with ataxia, bilateral facial weakness, diplopia, and oscillopsia. Thirteen days earlier, he had fever and progressive shortness of breath, followed by a productive cough and diarrhea (Wong et al. 2020). Headaches and dizziness are considered the non-specific minor symptom, associated with COVID-19 patients. The encephalopathy is reported in $40 \%$ of the patients (Mao et al. 2020a). Filatov et al. (Filatov et al. 2020) reported a case of a 74-year-old man with medical history of multiple diseases along with chronic pulmonary and Parkinson disease, who visited the emergency department of the hospital with cough and fever. The initial checkup did not represent any serious issues and he was sent home; later, he appeared again with a worsened condition and was tested COVID-19-positive. The Acute Hemorrhagic Necrotizing Encephalopathy (ANE) is developed because of the cytokine storm and causes disruption in the blood-brain barrier, and neuroinflammation which leads to the dysfunction of the brain.

The viral infections also trigger the "cytokine storm" which in response can lead nervous system damage. The cytokines are small chemically induced signaling molecules that produce healthy immune response. In a cytokine storm, the level of few cytokines soars farther than what is required, and in response, the immune cells of the body starts to strike healthy tissues. The ANE causes neuroinflammation and leads to dysfunction of the brain (Poyiadji et al. 2020).
Neuroinflammation caused by ANE resulting from cytokine storm characterized mainly by the increased production of the IL-6, which in turn have been activated by $\mathrm{T}$ helper cells, produced GM-CSF. Cytokine storm may also cause a surge in interleukins, interferons, monocytes, macrophage inflammatory proteins, and tumor necrosis factors leading to hyperinflammation. This systematic inflammation causes severe encephalopathy in the patient, which may leads to stroke (Mehta et al. 2020). The necrotizing hemorrhagic encephalopathy symptoms are endorsed from the CT scan images and MRI scan in COVID-19 patients. The immune-mediated events, either through the cytokine or chemokine pathways or via $\mathrm{T}$ cell, may ultimately lead to vascular leakage, demyelination, activation of complement and coagulation cascade, and end-organ damage (Natoli et al. 2020; Ahmad and Rathore 2020). The nervous system damage caused via viral infection and accompanied by the immune system is positively correlated with the severity of COVID-19 symptoms.

Poyiadji et al. (Poyiadji et al. 2020) reported the first case of COVID-19 associated with ANE from the USA. A female patient with 3 days history of cough, fever, and altered mental status appeared in a hospital. She was tested COVID-19-positive, and negative for Herpes simplex 1 and 2 virus. Later, the MRI scan of the patient brain demonstrated enhanced lesion in multiple regions, confirming the case of ANE.

During the COVID-19 infection, patients are likely to develop cerebrovascular accidents. Sharifi and colleagues (Sharifi-Razavi et al. 2020) reported a case of a 79-yearold COVID-19 patient, admitted in an emergency in a semi-conscious state, who had fever and cough in the past few days. The CT scan reports revealed a massive bleeding within the right hemisphere with the intraventricular and subarachnoid extension. The authors suggested that, maybe the dysregulation of ACE2 receptors leads to cerebral autoregulation, sympatho-adrenal system and cerebral blood flow could have resulted in the bleed. A Dutch patient infected with COVID-19 showed the abnormal clotting of blood. The blood clots can break and move to different parts of the body; it can even land in the lungs and block vital arteries, causing pulmonary embolism, which is reported as vital in COVID-19 patients. The blocking of arteries in the brain due to blood clots can cause stroke (Wadman et al. 2020).

Cytokine storms and hyperinflammatory responses can cause acute myelitis. Kang Zhao reported a case from Wuhan, a 66-year-old man who was admitted to a hospital with fever and body ache symptoms; later, he developed acute flaccid paralysis of the lower limbs and sensory level at T-10 with bowel and urinary uncontrollable leakage. Later, the PCR test confirmed the positive infection of COVID-19 in the patient along with patchy pneumonia observed via chest CT scans (Zhao et al. 2020a). 


\section{PNS manifestations}

The peripheral nervous system manifestation includes skeletal damage, anosmia, chemosensory dysfunction, and GuillainBarre Syndrome (GBS). Anosmia and chemosensory dysfunction were higher in the COVID-19 patients as compared with COVID-19-negative cases. A biologist, Alessandro Laurenzi, and many other COVID-19 patients in Italy complained about the loss of taste and smell (Yeager 2020). In a recent multicenter European study, 417 COVID-19 patients were studied, in which $85.6 \%$ patients had been diagnosed with olfactory dysfunction and $88.8 \%$ patients reported gustatory disorders. The otolaryngologist reported sudden anosmia or hyposmia as concurrent symptoms of COVID-19 infection. Among them, in $11.8 \%$ of patients, the olfactory dysfunction appeared before COVID-19 symptoms, $65.4 \%$ patients reported after COVID-19 symptoms, and $22.8 \%$ reported at the same time of COVID-19 general symptoms. Within the first 8 days, around $72.6 \%$ of patients recovered their olfactory functions (Lechien et al. 2020).

Mao et al. (Mao et al. 2020b), in his study of 214 Chinese, reported that $5.6 \%$ and $5.1 \%$ of patients showed impairment in taste and smell, respectively, though no taste dysfunction and anosmia are reported in COVID-19-positive patients of the French region.

So far, 8 cases of COVID-19 associated with GuillainBarre Syndrome (GBS) have been reported in China, Iran, and Italy. The first case of GBS reported was a 61-year-old woman in Wuhan City, China. The patient was admitted with acute weakness in her legs and severe fatigueness (Zhao et al. 2020b). Nerve conduction studies and electromyography showed nerve roots and peripheral nerve inflammation suggesting demyelinating polyneuropathy. The patient was tested COVID-19-positive, and after the treatment was provided, the patient showed a good motor recovery.

Sedaghat et al. (Sedaghat and Karimi 2020) reported a case from Iran, a 61-year-old diabetic patient who had fever, cough, and irregular dyspnea from two weeks before diagnosed with ascending paralysis leading to tetraplegia and bilateral facial paralysis. Acute motor and sensory axonal neuropathy were suggested after conducting the nerve conduction studies and electromyography studies.

Camdessanche et al. reported a 64-year-old man with 2 days history of cough and fever, who tested positive for COVID-19 and later developed paraesthesia in the hands and feet and progressive weakness in all the limbs on the 9th day of hospital admission, with areflexia and loss of vibration sense, then developed dysphagia and respiratory insufficiency (Camdessanche et al. 2020).

Virani and colleagues (Virani et al. 2020) reported a case, from the USA, of a 54-year-old male having GBS who was admitted to a hospital because of a rapidly progressing upper body paralysis leading to difficulty in respiration and later was tested positive for COVID-19. Since respiratory-related problems are common in COVID-19 patients and are also the probable reasons of GBS development in patients, the GBS should be considered a COVID-19 neurological complication.

The skeletal muscle injury reported in Mao et al.'s report shows about $19.3 \%$ of patients and $4.8 \%$ of patients in the severely ill and in the non-severe groups, respectively (Mao et al. 2020a). A report from China by Jin et al. reported a 60year-old man with COVID 19 who, 15 days later of onset, developed weakness and tenderness in the lower limbs (Jin and Tong 2020). It might be due to the effect of the virus directly on muscle tissues, or it could be the elevated response of cytokines in serum produced via infection-mediated immune response, resulting in damaging the skeletal muscle (Ahmad and Rathore 2020).

\section{Gene prioritization through disease-gene association data}

We have retrieved the list of 331 genes of humans known to interact with SARS-CoV-2 proteins from Gordan et al.'s study (Gordon et al. 2020). The gene-disease association data of 331 genes was retrieved from the GeneORGANizer database (Gokhman et al. 2017). GeneOrganizer database provides gene-disease, gene-phenotype, and gene expression region data of humans curated from human phenotype ontology (HPO) and DisGeNET tools. Out of 331 genes, 73 genes were showing their expression in the brain; their gene-disease and gene-phenotype associations were retrieved from the GeneOrganizer database. Genes having Human Phenotype Ontology (HPO) database-classified phenotypes were considered for further studies. Table 1 represents the interaction of COVID-19 proteins with the selected 73 genes. We constructed a protein-protein interaction network with the identified 73 genes using STRING plugin of a Cytoscape tool (Shannon et al. 2003); a network of 73 nodes and 81 edges were prepared (Fig. 2a). Gene ontology (GO) analysis of these 73 genes using DAVID (Sherman and Lempicki 2009) revealed that the genes are enriched in energy metabolism involving oxidation-reduction, nucleotide binding, electron carrier activity, and transport and regulation of neuronal apoptosis (Fig. 2b). The KEGG pathway enrichment analysis revealed the role of the genes in aminoacyl-tRNA biosynthesis.

Furthermore, we used Enrichr (Kuleshov et al. 2016), a web-based server for gene-set enrichment analysis and which provides different summaries of collective functions of gene lists. The disease enrichment analysis through DisGeNET (Pinero et al. 2020) revealed that the genes are mostly significantly associated with intellectual disability, development and cognitive delay, mental and motor retardation, and generalized hypotonia (Fig. 3a). Also, the enrichment through Jensen DISEASE (Pletscher-Frankild et al. 2015) analysis 
Table 1 COVID-19 targets expressed in the brain

\begin{tabular}{|c|c|c|}
\hline SARS-CoV-2 proteins & Genes & Count \\
\hline M protein & AASS, ACADM, ETFA, PMPCA, TARS2 & 5 \\
\hline $\mathrm{N}$ protein & RBM28, SNIP1 & 2 \\
\hline E protein & AP3B1, & 1 \\
\hline NSP1 & PKP2, POLA1 & 2 \\
\hline NSP4 & ALG11 & 1 \\
\hline NSP12 & BCKDK, TCF12 & 2 \\
\hline NSP7 & COMT, CYB5R3, DNAJC19, GNB1, MOGS, NDUFAF2, RAB18, RAB7A, RHOA, SCARB1 & 10 \\
\hline NSP8 & EXOXC3, EXOSC8, LARP7, NARS2, SEPSECS & 5 \\
\hline NSP9 & FBLN5, FBN1, FN2, MIB1, NUP62, POR & 6 \\
\hline NSP2 & GIGYF2 & 1 \\
\hline NSP14 & GLA & 1 \\
\hline NSP6 & SIGMAR1 & 1 \\
\hline NSP13 & TBK1, PRKACA, RDX, NIN, PCNT, GCC1, CEP135, CENPF, CDK5RAP2, AKAP9 & 10 \\
\hline ORF9c & ACAD9, BCS1L, NDUFAF1, NDUFB9, PIGO, WFS1 & 6 \\
\hline ORF9b & SLC9A3R1 & 1 \\
\hline ORF8 & $\begin{array}{l}\text { ADAM9, COL6A1, DNMT1, FKBP10, GDF15, IL17RA, ITGB1, LOX, MFGE8, NEU1, NGLY1, } \\
\text { NPC2, PLAT, SIL1, SMOC1, TOR1A, }\end{array}$ & 16 \\
\hline NSP5 & GPX1 & 1 \\
\hline ORF3a & HMOX1 & 1 \\
\hline ORF10 & PPT1 & 1 \\
\hline
\end{tabular}

showed that genes are highly associated with neurodegenerative disease, intellectual disability, and mitochondrial complex I deficiency (Fig. 3b). Overall, these results clearly showed that the gene signatures are largely involved in neurological manifestations following SARS-CoV-2 infection.

Network topological properties like degree of connectivity and betweenness centrality were calculated using a network analyzer plugin which showed seven genes with higher degree of connectivity and betweenness centrality value, and was considered hub genes. Hub genes are considered the most

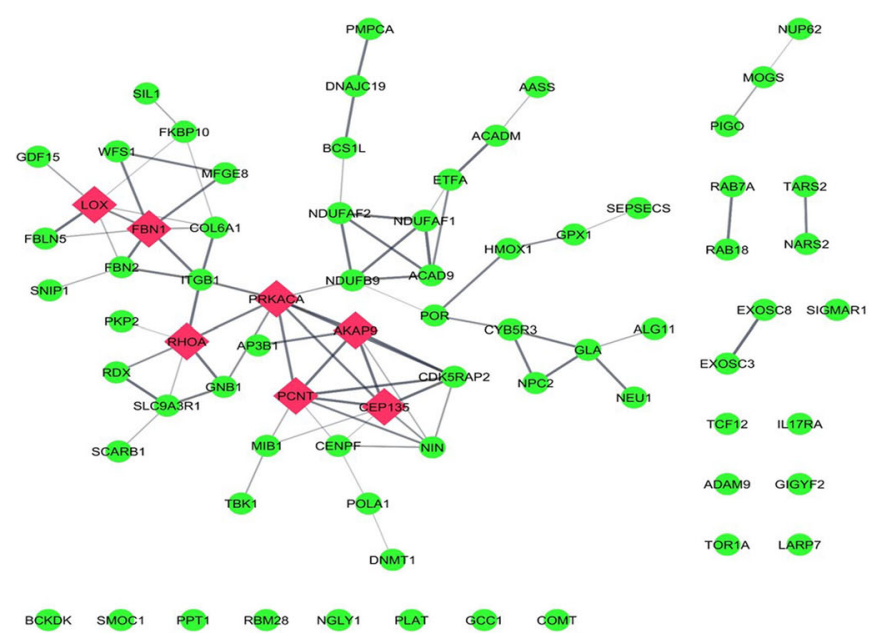

Fig. 2 (a) Protein-protein interaction (PPI) network of 73 genes from 331 human SARS-CoV-2 interacting genes showing relation to brain-related diseases using the gene-organizer database. Red color nodes represent the influential genes in controlling the network. The hub genes were LOX, FBN1, PRKACA, RHOA, PCNT, AKAP9, and CEP135. Many of these genes were found to interact with NSP13 of SARS-CoV-2 (PRKACA, CEP135, PCNT, and AKAP9) (Gordon et al. 2020). The NSP13 is a 596 amino acid-long, triangular pyramidal-shaped protein comprising 5 domains (Mirza and Froeyen 2020). It plays an important function in catalyzing the unwinding of duplex oligonucleotides into single strands in an NTP-dependent manner. The protein LOX interacts with ORF8 of SARS-CoV-2. The

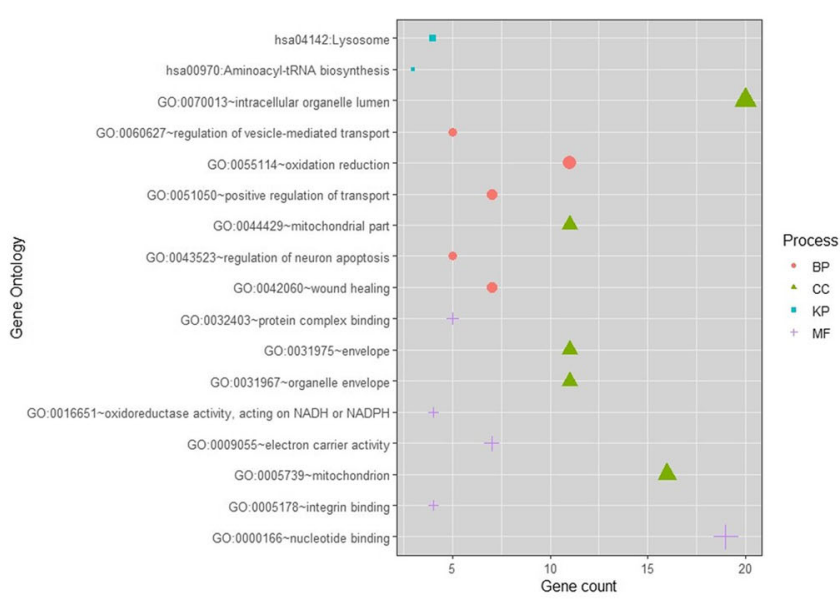

hub proteins in the network. (b) Functional enrichment analysis of genes through GO and KEGG analysis 

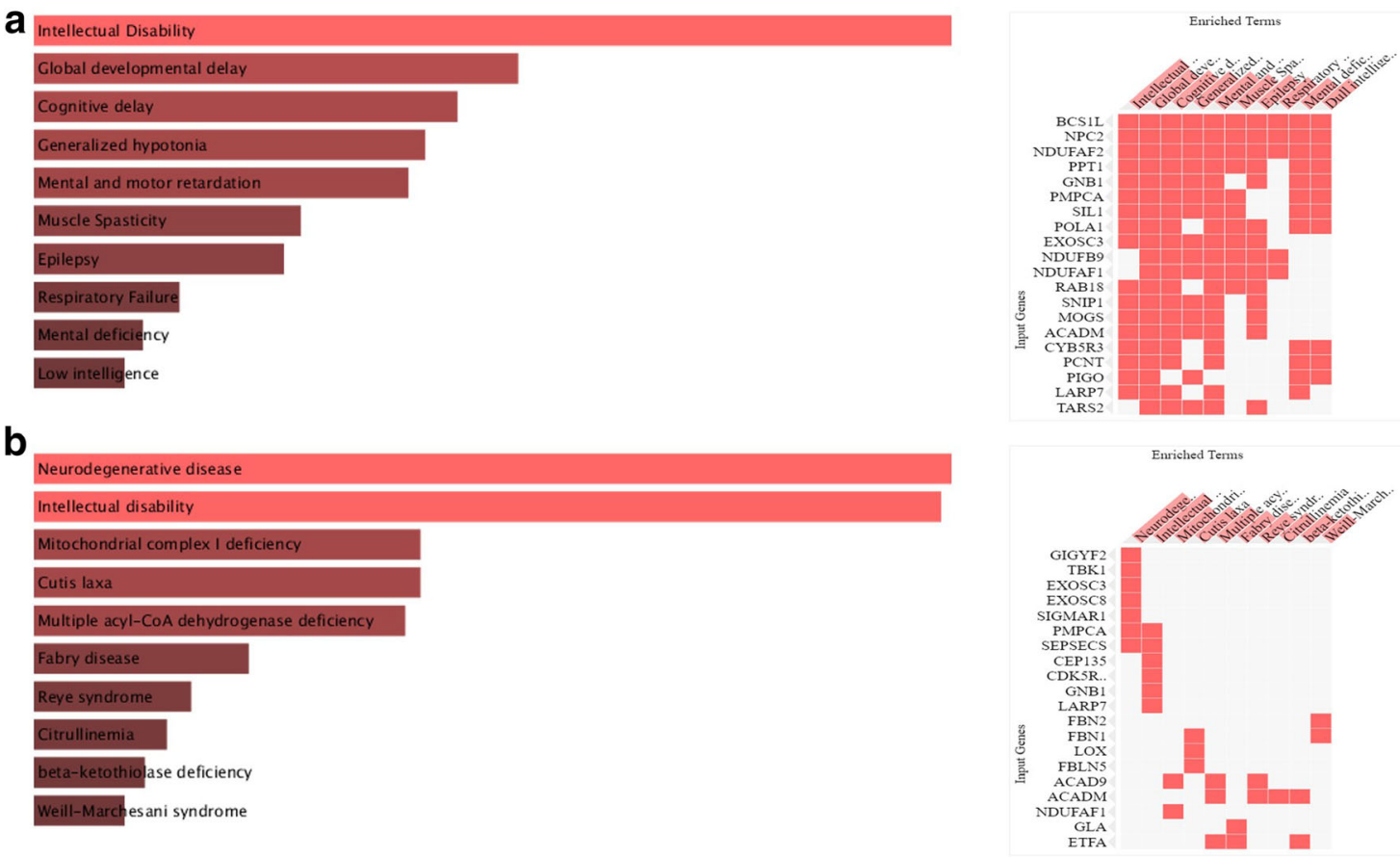

Fig. 3 Disease-based enrichment analysis through the Enrichr web server. Gene set enrichment analysis of top genes obtained through (a) DisGNet, and (b) Jensen DISEASE

ORF8 of SARS-CoV-2 is essential for viral replication. The drug CCT-365623 is an inhibitor of LOX with an IC50 of $0.89 \mu \mathrm{M}$ (Gordon et al. 2020). The protein RHOA interacts with NSP7 of SARS-CoV-2, while FBN1 interacts with NSP9 of SARS-CoV-2 (Gordon et al. 2020).

The human protein kinase cAMP-activated catalytic subunit alpha (PRKACA) encodes cyclic adenosine monophosphate (cAMP)-dependent kinase catalytic subunit alpha. In the human body, the PRKACA express in multiple tissues including the brain, skeletal muscles, and heart. The increase in the expression of PRKACA causes primary pigmented nodular adrenocortical disease (PPNAD). PPNAD is a rare condition and manifests as ACTH-independent Cushing's syndrome (CS). In this disease, patients suffer depression, emotional lability, mood changes, psychosis, anxiety, mental deterioration, agitation, diabetes mellitus, and hypercorticosolism (Carney and Young Jr 1992). Centrosomal protein of $135 \mathrm{kDa}$ (CEP135) is a conserved alpha-helical protein which is located at the centrosome throughout the cell cycle, and its localization is independent of the microtubule network. It plays an important role in centriole formation and elongation. Pericentrin (PCNT) protein is expressed in centrosome and is an integral part of the pericentriolar material (PCM). PCNT is involved in multiple roles including cilia formation, microtubule nucleation, and mitosis and as a centrosomal scaffold for multiple signaling proteins like PKA and PKC $\beta I I$ (Endoh-Yamagami et al. 2010). It is reported in multiple diseases including microcephaly often with mental retardation (Endoh-Yamagami et al.
2010). Fibrillin-1 (FBN1) is a structural macromolecule that polymerizes into microfibrils. It provides long-term force-bearing structural support. The symptoms in disease associated to FBN-1 also include obstructive sleep apnea and stroke. A kinase anchor protein 9 (AKAP9) is a large $450 \mathrm{kDa}$ scaffold protein. It is also known as centrosome and Golgi-localized protein kinase $\mathrm{N}$-associated protein (CG-NAP). It helps in the assembly of several protein kinases and phosphatases on the centrosome and the Golgi apparatus. The AKAP9 is needed to maintain the integrity of the Golgi apparatus (Witczak et al. 1999) and microtubular nucleation the cis-side of the Golgi apparatus (Larocca et al. 2004).

Ras homology family member A (RHOA) encodes for the Rho protein of GTPase family. It functions in signal transduction as molecular switches by cycling between the GDP-inactive and GTP-active bound states (Quilliam et al. 1996). The Rho family of GTPases plays an important role in various aspects of neuronal development such as neurite outgrowth and their differentiation, dendritic spine formation and their maintenance, and axon pathfinding. The activation of RHOA leads to the reorganization of actin cytoskeletal into distinct structures (Govek et al. 2005).

Lysyl oxidase (LOX) plays an important role in the formation and repair of the extracellular matrix by oxidizing lysine residues in the elastin and collagen, and in the stabilization of these fibrous proteins (Kumari et al. 2017). The LOX is associated to a number of diseases including hepatitis B, hepatitis $\mathrm{C}$, and Alzheimer's disease. Many COVID-19 patients 
reported stroke after the infection, as also reported in LOXassociated diseases.

\section{Gene prioritization through spatio-temporal expression analysis}

To study the spatio-temporal expression analysis of the selected 73 genes, we utilize the BEST (Brain Expression SpatioTemporal) web server (http://best.psych.ac.cn/\#) (Guo et al. 2019). This web server performs gene expression pattern analysis with reference to pre-integrated spatial-temporal expression data generated from healthy human brains. The server utilizes eight human brain expression datasets obtained from BrainSpan Atlas, Allen brainmap, GTEx, and other sources. To identify clusters of co-expressed genes, weighted gene correlation network analysis (WGCNA) was performed in five of the eight expression datasets.

The expression pattern of selected genes in different brain regions (spatial pattern) and age stages (temporal pattern) was analyzed using RNA-seq Data from Brainspan and RNA-seq Data from GTEx, and is shown in Fig. 4. As can be seen, most of the genes were upregulated from the neonatal stages to late adulthood (Fig. 4a, b). In older age, the genes were moderately upregulated in the cerebellum, hypothalamus, substantia nigra, hippocampus, and frontal cortex (Fig. 4b). The downregulation of these genes in the olfactory bulb, thalamus, and the cortical regions of the brain indicates the impairment of sensory systems, memory, and cognition.

Furthermore, the co-expression gene cluster enrichment analysis generated by WGCNA showed that these genes were enriched predominantly in neurons and in astrocytes to little extent (Fig. 4c). The most significantly enriched cluster is present in neurons in which the most significant gene is included. According to the expression heatmap (Fig. 4d), genes in this cluster are expressed in multiple brain regions and in the later stage of life. A protein-protein interaction network was then constructed using the genes in the enriched cluster (Fig. 4e). Among the 14 genes enriched in this cluster, the SNIP1 (Smad nuclear-interacting protein1) and ACADM (Acyl-CoA dehydrogenase, mitochondrial) gene has the most connections in the co-expression network (Fig. 4e). SNIP1 plays an important role in pre-mRNA splicing as a component of the spliceosome and is involved in the microRNA (miRNA) biogenesis. It also downregulates NF-kappa-B. On the other hand, ACADM is involved in mitochondrial fatty acid beta-oxidation and lipid metabolism.

\section{Gene prioritization by gene pathogenicity analysis}

We implemented gene prioritization using a novel framework, EvoTol (Rackham et al. 2015) to predict whether individual genes are likely to be disease-causing or not. The evolutionary intolerant study by EvoTol for the 73 genes showed that there are six genes whose average expression is $>100$ TPM in the central nervous system with $<50$ th percentile. Also, we identified robustly expressed genes for PNS, adult brain, and fetal brain (Fig. 5a). These putative pathogenic genes include CYB5R3, ITGB1, COL6A1, NPC2, RHOA, and RAB7A arranged according to the decrease in pathogenicity. As can be seen from the analysis, the protein CYB5R3 (cytochrome b5 reductase 3) is highly pathogenic and is less expressed in a

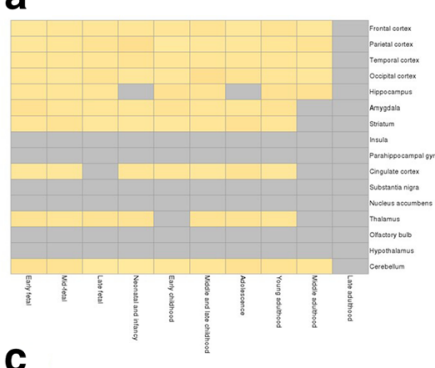

C

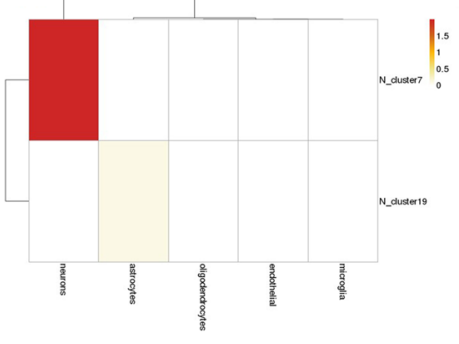

d

b

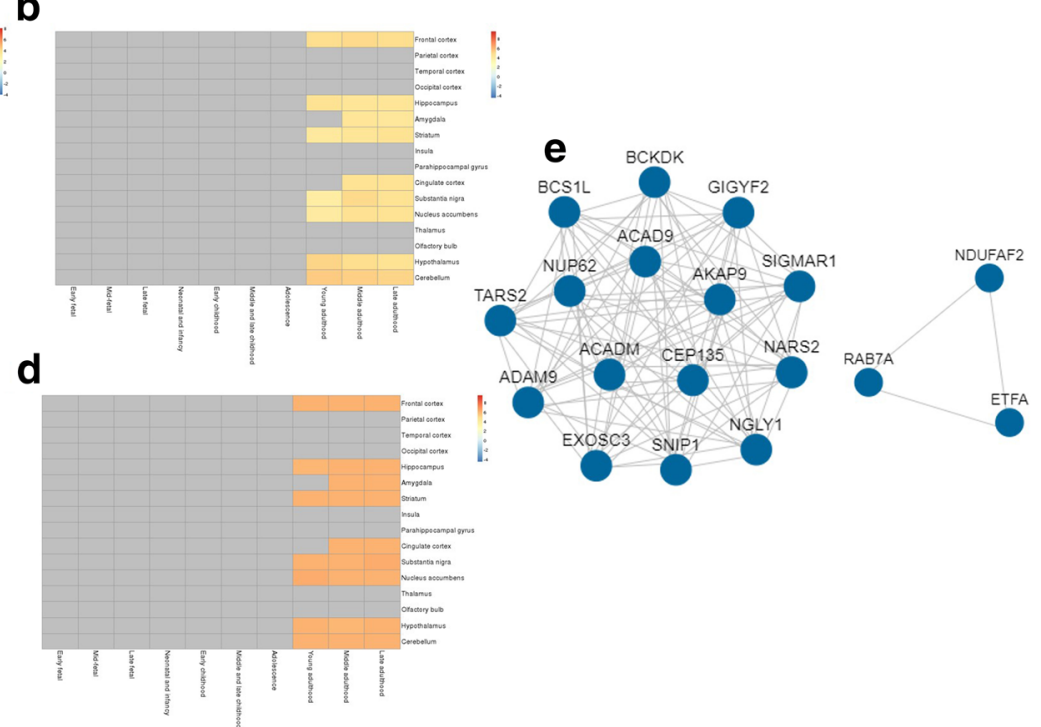

Fig. 4 BEST results of the 73 genes. Spatio-temporal expression heatmap of genes (a) RNA-Seq BrainSpan dataset, and (b) RNA-Seq GTEx dataset; (c) cell-type enrichment heatmap according to RNA-Seq GTEx; (d) expression heatmap of enriched clusters; and (e) core co-expression network of enriched genes 


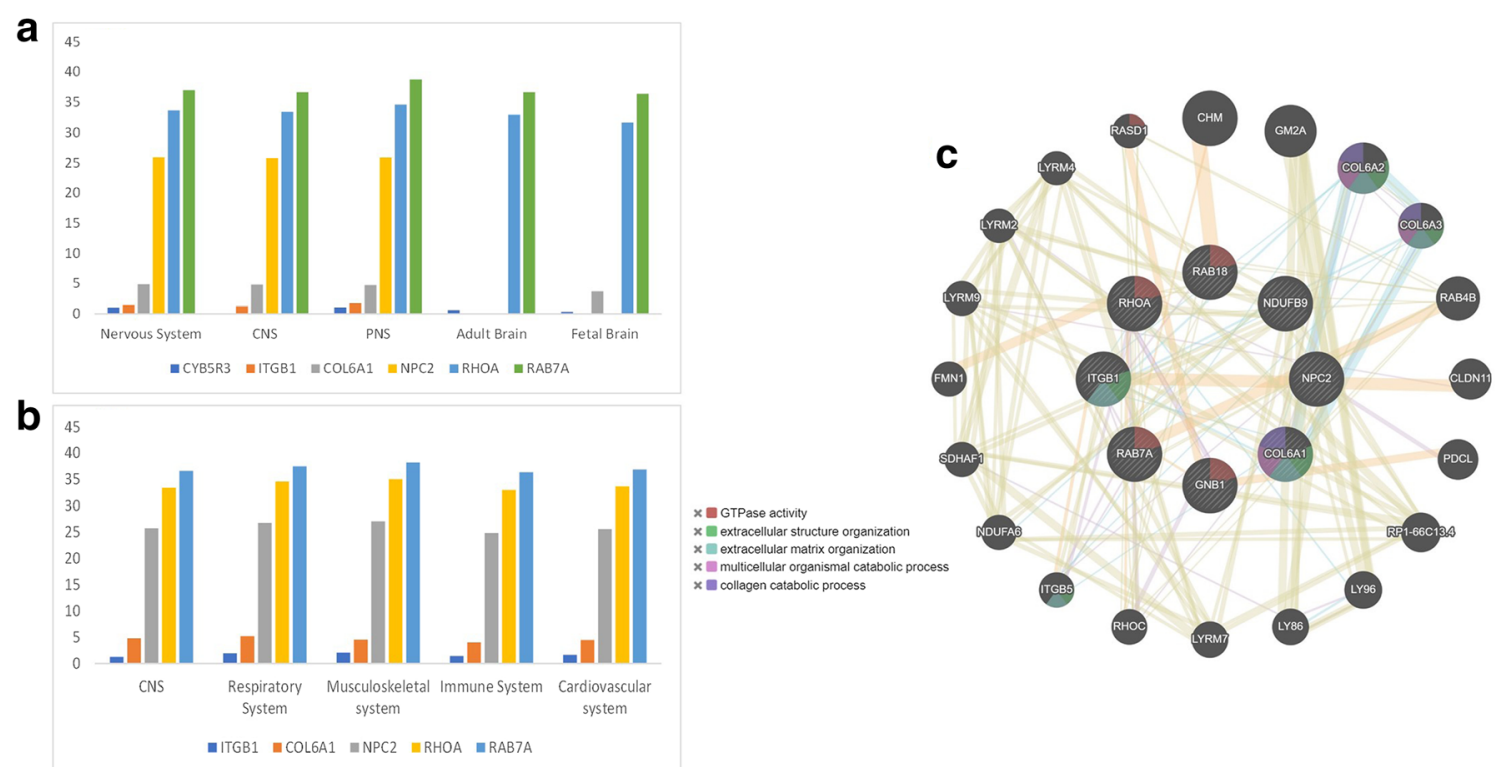

Fig. 5 Gene prioritization analysis through EvoTol. (a) The evolutionary intolerant gene expression in the nervous system and brain. (b) Enrichment of disease-causing genes in different human tissue-specific

CNS. The soluble isoform of CYB5R3 helps in the proper function of hemoglobin, while the membrane-bound isoform is involved in the breakdown and formation of fatty acids, the formation of cholesterol, and the breakdown of various molecules and drugs. It interacts with SARS-CoV-2 NSP7 and orf3 protein and hampers the electron transport process in human hosts (Gordon et al. 2020).

EvoTol can also be integrated with information on celltype and tissue-specific gene expression patterns to rank genes with respect to their pathogenicity in tissue-specific context. This information can prove useful to prioritize disease genes in disorders of the human system. We identified robustly expressed and most intolerant genes $(<50$ th percentile) in different human systems (Fig. 5b). We showed that these highly pathogenic genes are consistently expressed in all the systems, and these genes could explain the multi-system involvement in COVID-19.

Furthermore, to investigate whether these eight putative pathogenic genes were in a gene interaction network, we performed a network analysis using GeneMANIA (Warde-Farley et al. 2010) and obtained a protein interaction network (Fig. $5 c)$. The protein network is mainly enriched in GTPase activity and extracellular organization.

\section{Identification of genes interacting with COVID-19 receptors}

Recently, it has been established that spike protein of COVID-19 virus shows strong binding affinity with ACE2 and TMPRSS2 receptors and allows the virus to system. For each tissue-type, we identified robustly expressed genes as those whose average expression is > 100 TPM. (c) GeneMANIA network of the five putative pathogenic genes

enter the host's body (Zou et al. 2020). Therefore, genes interacting with ACE2 and TMPRSS2 receptors are significantly important, as after the virus-receptor interaction, these genes are the first one to receive the information. Simultaneously, any change in the expression of the genes directly interacting with COVID-19 receptors possibly might not allow the binding of virus to the receptors and can serves as a potential therapeutic target. We retrieved the list of genes interacting with ACE2 and TMPRSS2 receptors from multiple literatures, uniport database, and string database (Wicik et al. 2020; Consortium 2019; Szklarczyk et al. 2015). Out of 73 genes showing their expression in brain, we identified that a gene Integrin beta-1 (ITGB1) has a direct interaction with ACE2 receptor (Fig. 6a). ITGB1 are known to bind with ACE2 receptor and not with ACE receptors. ACE2 has a highly conserved binding domain for integrin in their ectodomain (Clarke et al. 2012). The ITGB1 gene plays a role in many important pathways like ECM-receptor interaction, proteoglycans in cancer, cell adhesion molecules, Axon guidance, Rap1-signaling pathway, and PI3K-AKT signaling pathways (Kanehisa et al. 2016). Furthermore, to explore the temporal expression patterns of ITGB1, we used the Human Brain Transcriptome project (HBT; http://hbatlas.org/pages/hbtd). The HBT project provides transcriptome data for the developing and adult human brain. As can be seen in Fig. 6b, ITGB1 expression appeared to be decreased in early childhood and after that remained stable over adulthood and older age. The examined six brain regions include the hippocampus, amygdala, striatum, cerebellar cortex, mediodorsal nucleus of the thalamus, and neocortex. 
Fig. 6 (a) Interaction of 73 genes with COVID-19 receptors.

ITGB1 gene showing interaction with ACE2 receptor (square shaped) along with 3 hubs genes of the network (hexagon-shaped). (b) ITGB1 expression levels across the six brain regions over the human life span are shown as obtained from HBT

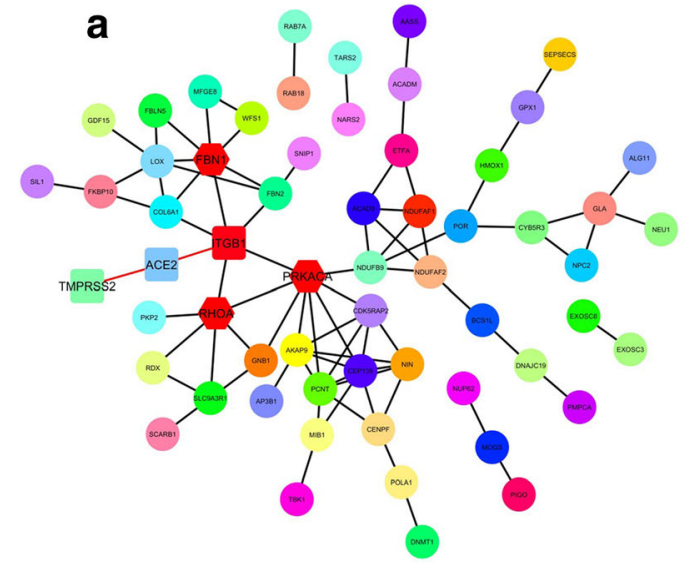

ITGB1 also shows association with many diseases such as neural tube defects, sarcoma, psoriasis, cerebral hypoxia, lymphoma, and leukemia and are also associated with multiple cancers (Stelzer et al. 2016). ITGB1 also regulated the myelination of nerves, which is an important process as myelination insulates the nerves and increases the rate of information flow and are required for normal CNS myelination (Barros et al. 2009). We also observed that ITGB1 in our network is also interacting with three of the hub genes (namely FBN1, PRKACA, and RHOA) (hexagon-shaped in red), which means any information received by ITGB1 because of the virus-receptor binding will transfer to these hub genes which they eventually transfer to the major part of the network as they are well-connected genes in the network leading to possible multiple neurological disorders.

\section{Conclusion}

The underlying pathophysiology of neurological manifestations in COVID-19 remains to be fully understood. Despite an increasing number of studies in that field, more data are required to satisfactorily correlate these two and their clinical impact. The physicians should consider, apart from respiratory symptoms, the neurological symptoms like encephalitis, seizures, twitching, and loss of taste and smell. The longterm effect of the neuroinvasive nature of COVID-19 may increase the risk of neurodegenerative disease like multiple sclerosis. In this study, we have identified 73 human targets of SARS-CoV-2 according to their association with brainrelated diseases. Through gene prioritization according to the spatio-temporal expression in the brain and through evolutionary intolerance analysis, we have identified putative pathogenic genes. Through network analysis, we have identified ITGB1 as an ACE2 binding protein and is highly expressed in the brain. This protein could be considered a drug target for the prevention and treatment of CNS symptoms associated with COVID-19 patients.
Acknowledgments The authors sincerely thank the Amity University, Noida, for providing facilities.

\section{Compliance with ethical standards}

Conflict of interest The authors declare that they have no conflict of interest.

\section{References}

Ahmad I, Rathore FA (2020) Neurological manifestations and complications of COVID-19: a literature review. J Clin Neurosci 77:8-12

Baig, A.M., et al., Evidence of the COVID-19 virus targeting the CNS: tissue distribution, host-virus interaction, and proposed neurotropic mechanisms. ACS chemical neuroscience, 2020.

Barros CS, Nguyen T, Spencer KSR, Nishiyama A, Colognato H, Muller $\mathrm{U}$ (2009) $\beta 1$ integrins are required for normal CNS myelination and promote AKT-dependent myelin outgrowth. Development 136(16): 2717-2724

Bohmwald K, Gálvez NMS, Ríos M, Kalergis AM (2018) Neurologic alterations due to respiratory virus infections. Front Cell Neurosci 12:386

Camdessanche J-P et al (2020) COVID-19 may induce Guillain-Barré syndrome. Rev Neurol

Carney JA, Young WF Jr (1992) Primary pigmented nodular adrenocortical disease and its associated conditions. Endocrinologist 2(1):6-21

Clarke NE, Fisher MJ, Porter KE, Lambert DW, Turner AJ (2012) Angiotensin converting enzyme (ACE) and ACE2 bind integrins and ACE2 regulates integrin signalling. PLoS One 7(4):e34747

Consortium U (2019) UniProt: a worldwide hub of protein knowledge. Nucleic Acids Res 47(D1):D506-D515

Ellul, M., et al., Neurological associations of COVID-19. Available at SSRN 3589350, 2020.

Endoh-Yamagami S, Karkar KM, May SR, Cobos I, Thwin MT, Long JE, Ashique AM, Zarbalis K, Rubenstein JLR, Peterson AS (2010) A mutation in the pericentrin gene causes abnormal interneuron migration to the olfactory bulb in mice. Dev Biol 340(1):41-53

Filatov A et al (2020) Neurological complications of coronavirus disease (COVID-19): encephalopathy. Cureus 12(3)

Gklinos P (2020) Neurological manifestations of COVID-19: a review of what we know so far. J Neurol 267:2485-2489

Gokhman D, Kelman G, Amartely A, Gershon G, Tsur S, Carmel L (2017) Gene ORGANizer: linking genes to the organs they affect. Nucleic Acids Res 45(W1):W138-W145 
Gordon DE, Jang GM, Bouhaddou M, Xu J, Obernier K, White KM, O'Meara MJ, Rezelj VV, Guo JZ, Swaney DL, Tummino TA, Hüttenhain R, Kaake RM, Richards AL, Tutuncuoglu B, Foussard H, Batra J, Haas K, Modak M, Kim M, Haas P, Polacco BJ, Braberg H, Fabius JM, Eckhardt M, Soucheray M, Bennett MJ, Cakir M, McGregor MJ, Li Q, Meyer B, Roesch F, Vallet T, Mac Kain A, Miorin L, Moreno E, Naing ZZC, Zhou Y, Peng S, Shi Y, Zhang Z, Shen W, Kirby IT, Melnyk JE, Chorba JS, Lou K, Dai SA, Barrio-Hernandez I, Memon D, HernandezArmenta C, Lyu J, Mathy CJP, Perica T, Pilla KB, Ganesan SJ, Saltzberg DJ, Rakesh R, Liu X, Rosenthal SB, Calviello L, Venkataramanan S, Liboy-Lugo J, Lin Y, Huang XP, Liu YF, Wankowicz SA, Bohn M, Safari M, Ugur FS, Koh C, Savar NS, Tran QD, Shengjuler D, Fletcher SJ, O'Neal MC, Cai Y, Chang JCJ, Broadhurst DJ, Klippsten S, Sharp PP, Wenzell NA, Kuzuoglu-Ozturk D, Wang HY, Trenker R, Young JM, Cavero DA, Hiatt J, Roth TL, Rathore U, Subramanian A, Noack J, Hubert M, Stroud RM, Frankel AD, Rosenberg OS, Verba KA, Agard DA, Ott M, Emerman M, Jura N, von Zastrow M, Verdin E, Ashworth A, Schwartz O, d'Enfert C, Mukherjee S, Jacobson M, Malik HS, Fujimori DG, Ideker T, Craik CS, Floor SN, Fraser JS, Gross JD, Sali A, Roth BL, Ruggero D, Taunton J, Kortemme T, Beltrao P, Vignuzzi M, García-Sastre A, Shokat KM, Shoichet BK, Krogan NJ (2020) A SARS-CoV-2 protein interaction map reveals targets for drug repurposing. Nature 583:459-468

Govek E-E, Newey SE, Van Aelst L (2005) The role of the Rho GTPases in neuronal development. Genes Dev 19(1):1-49

Guo L, Lin W, Zhang Y, Li W, Wang J (2019) BEST: a web server for brain expression Spatio-temporal pattern analysis. BMC Bioinf 20(1):632

Helms J, Kremer S, Meziani F (2020a) More on neurologic features in severe SARS-CoV-2 infection. Reply N Engl J Med 382

Helms J, Kremer S, Merdji H, Clere-Jehl R, Schenck M, Kummerlen C, Collange O, Boulay C, Fafi-Kremer S, Ohana M, Anheim M, Meziani F (2020b) Neurologic features in severe SARS-CoV-2 infection. N Engl J Med 382(23):2268-2270

Jin M, Tong Q (2020) Rhabdomyolysis as potential late complication associated with 2019 novel coronavirus disease. Emerg Infect Dis 26

Kanehisa M, Sato Y, Kawashima M, Furumichi M, Tanabe M (2016) KEGG as a reference resource for gene and protein annotation. Nucleic Acids Res 44(D1):D457-D462

Kuleshov MV, Jones MR, Rouillard AD, Fernandez NF, Duan Q, Wang Z, Koplev S, Jenkins SL, Jagodnik KM, Lachmann A, McDermott MG, Monteiro CD, Gundersen GW, Ma'ayan A (2016) Enrichr: a comprehensive gene set enrichment analysis web server 2016 update. Nucleic Acids Res 44(W1):W90-W97

Kumari S, Panda TK, Pradhan T (2017) Lysyl oxidase: its diversity in health and diseases. Indian J Clin Biochem 32(2):134-141

Lahiri, D. and A. Ardila, COVID-19 pandemic: a neurological perspective. Cureus, 2020

Larocca MC, Shanks RA, Tian L, Nelson DL, Stewart DM, Goldenring JR (2004) AKAP350 interaction with cdc42 interacting protein 4 at the Golgi apparatus. Mol Biol Cell 15(6):2771-2781

Lechien JR et al (2020) Olfactory and gustatory dysfunctions as a clinical presentation of mild-to-moderate forms of the coronavirus disease (COVID-19): a multicenter European study. Eur Arch Otorhinolaryngol:1-11

Leonardi M, Padovani A, McArthur JC (2020) Neurological manifestations associated with COVID-19: a review and a call for action. J Neurol 267:1573-1576

Li, Y.C., W.Z. Bai, and T. Hashikawa, The neuroinvasive potential of SARS-CoV2 may play a role in the respiratory failure of COVID-19 patients. J Med Virol, 2020.
Mao, L., et al., Neurologic manifestations of hospitalized patients with coronavirus disease 2019 in Wuhan, China. JAMA neurology, 2020a.

Mao, L., et al., Neurologic Manifestations of hospitalized patients with coronavirus disease 2019 in Wuhan, China. JAMA Neurol, 2020b

McGavern DB, Kang SS (2011) Illuminating viral infections in the nervous system. Nat Rev Immunol 11(5):318-329

Mehta P, McAuley D, Brown M, Sanchez E, Tattersall RS, Manson JJ, HLH Across Speciality Collaboration, UK (2020) COVID-19: consider cytokine storm syndromes and immunosuppression. Lancet 395(10229): 1033-1034

Mirza, M.U. and M. Froeyen, Structural elucidation of SARS-CoV-2 vital proteins: computational methods reveal potential drug candidates against main protease, Nsp12 polymerase and Nsp13 helicase. J Pharm Anal, 2020.

Moriguchi T, Harii N, Goto J, Harada D, Sugawara H, Takamino J, Ueno M, Sakata H, Kondo K, Myose N, Nakao A, Takeda M, Haro H, Inoue O, Suzuki-Inoue K, Kubokawa K, Ogihara S, Sasaki T, Kinouchi H, Kojin H, Ito M, Onishi H, Shimizu T, Sasaki Y, Enomoto N, Ishihara H, Furuya S, Yamamoto T, Shimada S (2020) A first case of meningitis/encephalitis associated with SARS-Coronavirus-2. Int J Infect Dis 94:55-58

Natoli, S., et al., Does SARS-Cov-2 invade the brain? Translational lessons from animal models. Eur J Neurol, 2020.

Paniz-Mondolfi A, Bryce C, Grimes Z, Gordon RE, Reidy J, Lednicky J, Sordillo EM, Fowkes M (2020) Central nervous system involvement by severe acute respiratory syndrome coronavirus-2 (SARSCoV-2). J Med Virol 92(7):699-702

Pinero J et al (2020) The DisGeNET knowledge platform for disease genomics: 2019 update. Nucleic Acids Res 48(D1):D845-D855

Pletscher-Frankild S, Pallejà A, Tsafou K, Binder JX, Jensen LJ (2015) DISEASES: text mining and data integration of disease-gene associations. Methods 74:83-89

Poyiadji, N., et al., COVID-19-associated acute hemorrhagic necrotizing encephalopathy: CT and MRI features. Radiology, 2020: p. 201187.

Quilliam LA, Lambert QT, Mickelson-Young LA, Westwick JK, Sparks AB, Kay BK, Jenkins NA, Gilbert DJ, Copeland NG, der CJ (1996) Isolation of a NCK-associated kinase, PRK2, an SH3-binding protein and potential effector of Rho protein signaling. J Biol Chem 271(46):28772-28776

Rackham OJ et al (2015) EvoTol: a protein-sequence based evolutionary intolerance framework for disease-gene prioritization. Nucleic Acids Res 43(5):e33

Rogers, J.P., et al., Psychiatric and neuropsychiatric presentations associated with severe coronavirus infections: a systematic review and meta-analysis with comparison to the COVID-19 pandemic. Lancet Psychiatry, 2020.

Sedaghat Z, Karimi N (2020) Guillain Barre syndrome associated with COVID-19 infection: a case report. J Clin Neurosci 76:233-235

Shannon P, Markiel A, Ozier O, Baliga NS, Wang JT, Ramage D, Amin N, Schwikowski B, Ideker T (2003) Cytoscape: a software environment for integrated models of biomolecular interaction networks. Genome Res 13(11):2498-2504

Sharifi-Razavi A, Karimi N, Rouhani N (2020) COVID-19 and intracerebral haemorrhage: causative or coincidental? New Microbes New Infect 35:100669

Sherman BT, Lempicki RA (2009) Systematic and integrative analysis of large gene lists using DAVID bioinformatics resources. Nat Protoc 4(1):44

Stelzer G et al (2016) The GeneCards suite: from gene data mining to disease genome sequence analyses. Curr Protoc Bioinformatics 54(1):1.30. 1-1.30. 33

Szklarczyk D, Franceschini A, Wyder S, Forslund K, Heller D, HuertaCepas J, Simonovic M, Roth A, Santos A, Tsafou KP, Kuhn M, Bork P, Jensen LJ, von Mering C (2015) STRING v10: protein- 
protein interaction networks, integrated over the tree of life. Nucleic Acids Res 43(D1):D447-D452

Thompson R (2020) Pandemic potential of 2019-nCoV. Lancet Infect Dis 20(3): 280

Toljan K (2020) Letter to the editor regarding the viewpoint "Evidence of the COVID-19 virus targeting the cns: tissue distribution, host-virus interaction, and proposed Neurotropic mechanism”. ACS Chem Neurosci 11(8): 1192-1194

Troyer, E.A., J.N. Kohn, and S. Hong, Are we facing a crashing wave of neuropsychiatric sequelae of COVID-19? Neuropsychiatric symptoms and potential immunologic mechanisms. Brain Behav Immun, 2020.

Varatharaj, A., Thomas N., Ellul M., Davies N.W.S., Pollak T., Tenorio E.L., Sultan M., Easton A., Breen G., Zandi M., Coles J.P., Manji H., al-Shahi Salman R., Menon D., Nicholson T., Benjamin L., Carson A., Smith C., Turner M.R., Solomon T., Kneen R., Pett S., Galea I., Thomas R.H., Michael B., UK-wide surveillance of neurological and neuropsychiatric complications of COVID-19: the first 153 patients. Available at SSRN 2020. https://doi.org/10.2139/ssrn. 3601761.

Virani A, Rabold E, Hanson T, Haag A, Elrufay R, Cheema T, Balaan M, Bhanot N (2020) Guillain-Barré syndrome associated with SARSCoV-2 infection. IDCases 20:e00771

Wadman, M., et al., A rampage through the body. 2020, Am Assoc Adv Sci

Wang D, Hu B, Hu C, Zhu F, Liu X, Zhang J, Wang B, Xiang H, Cheng Z, Xiong Y, Zhao Y, Li Y, Wang X, Peng Z (2020) Clinical characteristics of 138 hospitalized patients with 2019 novel coronavirusinfected pneumonia in Wuhan, China. Jama 323(11):1061-1069

Warde-Farley D et al (2010) The GeneMANIA prediction server: biological network integration for gene prioritization and predicting gene function. Nucleic Acids Res 38(Web Server issue):W214-W220

Whittaker A, Anson M, Harky A (2020) Neurological manifestations of COVID-19: a systematic review and current update. Acta Neurol Scand 142(1):14-22

Wicik, Z., et al., ACE2 interaction networks in COVID-19: a physiological framework for prediction of outcome in patients with cardiovascular risk factors. BioRxiv, 2020.

Witczak O, Skålhegg BS, Keryer G, Bornens M, Taskén K, Jahnsen T, Orstavik S (1999) Cloning and characterization of a cDNA encoding an A-kinase anchoring protein located in the centrosome, AKAP450. EMBO J 18(7):1858-1868
Wong PF, Craik S, Newman P, Makan A, Srinivasan K, Crawford E, Dev D, Moudgil H, Ahmad N (2020) Lessons of the month 1: a case of rhombencephalitis as a rare complication of acute COVID-19 infection. Clin Med 20(3):293-294

Wrapp D, Wang N, Corbett KS, Goldsmith JA, Hsieh CL, Abiona O, Graham BS, McLellan JS (2020) Cryo-EM structure of the 2019$\mathrm{nCoV}$ spike in the prefusion conformation. Science 367(6483): 1260-1263

Wu D, Yang XO (2020) TH17 responses in cytokine storm of COVID19: an emerging target of JAK2 inhibitor Fedratinib. J Microbiol Immunol Infect 53:368-370

Wu F, Zhao S, Yu B, Chen YM, Wang W, Song ZG, Hu Y, Tao ZW, Tian JH, Pei YY, Yuan ML, Zhang YL, Dai FH, Liu Y, Wang QM, Zheng JJ, Xu L, Holmes EC, Zhang YZ (2020) A new coronavirus associated with human respiratory disease in China. Nature 579(7798):265-269

Xiang P et al (2020) First case of 2019 novel coronavirus disease with encephalitis. ChinaXiv T202003:00015

Xu Z, Shi L, Wang Y, Zhang J, Huang L, Zhang C, Liu S, Zhao P, Liu H, Zhu L, Tai Y, Bai C, Gao T, Song J, Xia P, Dong J, Zhao J, Wang FS (2020) Pathological findings of COVID-19 associated with acute respiratory distress syndrome. Lancet Respir Med 8(4):420-422

Yan R, Zhang Y, Li Y, Xia L, Guo Y, Zhou Q (2020) Structural basis for the recognition of SARS-CoV-2 by full-length human ACE2. Science 367(6485): 1444-1448

Yeager, A., Lost smell and taste hint COVID-19 can target the nervous system. The Scientist, 2020.

Kang Zhao, et al., Acute myelitis after SARS-CoV-2 infection: a case report. medRxiv, 2020a. https://doi.org/10.1101/2020.03.16. 20035105 .

Zhao H, Shen D, Zhou H, Liu J, Chen S (2020b) Guillain-Barré syndrome associated with SARS-CoV-2 infection: causality or coincidence? Lancet Neurol 19(5):383-384

Zou X et al (2020) Single-cell RNA-seq data analysis on the receptor ACE2 expression reveals the potential risk of different human organs vulnerable to 2019-nCoV infection. Front Med 14:1-8

Publisher's note Springer Nature remains neutral with regard to jurisdictional claims in published maps and institutional affiliations. 